\title{
Deciphering the new global initiative for chronic obstructive lung disease (GOLD) guideline
}

\author{
Elvis M. Irusen
}

Published online: 30 June 2012

(C) Springer Science+Business Media, LLC 2012

\begin{abstract}
Major research into molecular mechanisms and recently performed large-scale clinical studies in COPD have led to important insights into the pathogenesis and the clinical course of COPD. Based on these, the new GOLD guide has been completely revised. Certain pillars of management e.g. smoking cessation, pulmonary rehabilitation, and identifying and managing co-morbidities have been reinforced. The major change is the move away from the old staging system (which was more generalized and based largely on lung function deficit) towards the categorization of patients into 4 groups designated A, B, C, and D using three composite measures. These measures quantify the severity of lung function impairment, the degree of breathlessness, and the history of exacerbations. They now allow for a more individualized approach based on symptoms and future risk of adverse events and exacerbations. Mild degrees of dyspnea and lung function impairment and occasional exacerbations place subjects in the low-risk categories (A\&B). More severe dyspnea and lung function impairment and a history of exacerbations $\geq 2$ per year would lead to the categorization of higher risk (C\&D). Low-risk patients are managed with bronchodilator therapies but high-risk patients are best managed with long-acting bronchodilators alone or a combination of bronchodilator classes, with or without inhaled corticosteroids. A novel phosphodiesterase inhibitor, roflumilast, is also an add-on alternative in high-risk patients. Utilizing these strategies will allow for improved clinical outcomes with respect to symptom relief and quality of life and additionally, in high-risk groups, reduce the chances of disease progression, exacerbations, and possible mortality.
\end{abstract}

\section{E. M. Irusen $(\triangle)$}

Division of Pulmonology, Department of Medicine, Faculty of Medicine and Health Sciences, University of Stellenbosch, P O Box 19063, Cape Town, South Africa

e-mail: eirusen@sun.ac.za
Keywords GOLD · COPD · Breathlessness · Airflow limitation $\cdot$ Spirometry $\cdot$ Exacerbations

\section{Introduction}

The new COPD guideline advocated by GOLD might appear at first glance to be confusing but is in fact the conceptualization of the modern approach in therapeutics applied to COPD [1••]. This view looks beyond simple pharmacological mechanisms of drug action but towards patient centered outcomes as the goals of therapy as the disease is phenotypically heterogenous. This phase represents the succession to the other major obstructive lung disease-asthma.

The launch of molecular medicine initially found fertile territory in unlocking the mechanisms of allergic sensitization and pathogenetic sequelae of asthmatic inflammation. Pari passu were the important and recurring clinical unmet needs - major morbidity and unnecessary mortality [2•]. The development of potent and safer inhaled corticosteroids and long-acting $\beta_{2}$ agonists and improved inhaler devices resulted in effective and convenient medication becoming available to increased numbers of patients. The clinical surveys underscored the ongoing need for education of both patients and practitioners to ensure complete control of airway inflammation and thus impacting on patient centered outcomes: amelioration of symptoms, decreased exacerbation risk, and the preservation of lung function and structure [3]. A number of large scale clinical trials attested to this reality $[4 \cdot, 5]$. With this clearer appreciation of asthma, the focus and pace of research on COPD increased; molecular mechanisms were unravelled and large scale trials embarked upon $[6,7 \bullet \bullet, 8]$. These have provided scientific clarity with regards to pathogenetic mechanisms and clinical utility regarding COPD outcomes. Select aspects will now be discussed and the rationale of the composite evaluation and 
appropriate therapeutic options in the new GOLD guideline will be explained. This approach makes the assessment and management much more personalized than the previous guide which was more generalized and based largely on lung function impairment.

\section{Expanded clinical outcomes in COPD}

The expanded clinical outcomes in COPD encompass the following and represent the expectation of existing and future drug therapy:

1. Symptom relief from sustained bronchodilatation

2. Decreased pulmonary inflammation

3. Prevention of continual lung damage and lung function decline

4. Decrease exacerbation risk

5. Improvement in functional capacity

6. Decrease in mortality

Symptom relief from sustained bronchodilatation

Airflow limitation due to narrowed airways is the hallmark of the major obstructive lung disorders. There are, however, two fundamental differences.

Firstly in asthma, bronchoconstriction is episodic whilst in COPD it is constant. Secondly and as a consequence of the latter, the ability of the lungs to empty (as reflected in the residual volume or air trapping) is affected to a much greater degree in COPD. The ensuing hyperinflation of the lung impairs the inspiratory capacity. Thus both expiratory limitation and impaired inspiration accentuate the respiratory effort and increase the work of breathing; these are perceived as breathlessness.

Patients with mild symptoms benefit from short acting $\beta_{2}$ agonists (SABA) e.g. albuterol or short acting muscarinic antagonists (SAMA) e.g. ipratropium bromide. However, because of the aforementioned pathophysiology, long acting $\beta_{2}$ agonists (LABA) e.g. salmeterol, formoterol, and indacaterol or long acting muscarinic antagonists (LAMA) e.g. tiotropium bromide are superior so as to allow for both bronchodilatation and lung deflation, especially if there are frequent symptoms. Additionally inhaled corticosteroids (ICS) augment the action of the bronchodilators improving lung function, symptoms, and quality of life.

It is perhaps prudent at this juncture to clarify a major misconception regarding bronchodilator responses in COPD. GOLD initially defined COPD as "not fully reversible" one must remember that this referred to the bronchodilator response (BDR) that did not normalize as determined spirometrically, although there can be significant bronchodilatation. Additionally, the American Thoracic Society and
European Respiratory Society have defined a positive BDR or reversible airflow obstruction - the most frequently cited being FEV1 or FVC improvement after albuterol of $\geq 12 \%$ and $\geq 200 \mathrm{~mL}[9,10]$. However, this has been misconstrued to imply a diagnostic label i.e. that a positive BDR would be synonymous with asthma - this is patently untrue. Using the aforementioned numeric criteria COPD can be reversibleand to quite a significant extent $[11,12 \bullet, 13]$. Since the first GOLD document, practitioners have been using the BDR to decide whether a subject has asthma or COPD, frequently ignoring the clinical information. Furthermore, in almost every clinical trial in the last decade, the American and European drug regulatory authorities have insisted that COPD patients recruited do not show a positive BDR, i.e. the FEV1 should be $\leq 12 \%$ and $200 \mathrm{~mL}$.

One determines whether a patient has COPD or asthma after the clinical assessment and does not let spirometry dictate the diagnosis - it is supplementary. To iterate, COPD can be reversible and similarly, asthma can be "irreversible" on the BDR, in the latter, when uncontrolled inflammation inhibits adequate bronchodilatation.

It is also counterintuitive to consider COPD "irreversible" and yet the mainstay of therapy is the bronchodilators that reverse airway bronchoconstriction.

\section{Decreased pulmonary inflammation}

Many of the inflammatory pathways involved in COPD have been elucidated but the development of antiinflammatory agents specific for COPD has been difficult.

\section{Inhaled corticosteroids}

Physicians are most familiar with corticosteroids which are very effective in asthmatic inflammation. Unfortunately, in COPD, most inflammatory pathways are steroid resistant.

Many clinicians and scientists believe that ICS are ineffective and their use is considered quite controversial [14]. There is some merit because a number of in vitro experiments appeared not to show significant attenuation of COPD inflammation. However, an error was made in studying and likening COPD inflammation to asthmatic inflammation; in the latter improvements are seen quite rapidly and when these findings could not be replicated in COPD, ICS were considered ineffective. Longer term studies, by contrast, have demonstrated a range of anti-inflammatory properties that may well contribute to the beneficial effects [15-17].

There have been some concerns over ICS safety. However, even when used in very high doses (up to $2000 \mu \mathrm{g}$ beclomethasone equivalent) they have been exceptionally safe: there has been no increase in skin bruising, cataracts, osteoporosis, or fractures. It must be remembered that 
smoking, advanced age and oral steroids are much more deleterious in these patients and are risk factors in their own right. For example, in the Towards a Revolution in COPD Health (TORCH) study, $18 \%$ of men and $30 \%$ of women had osteoporosis, and $42 \%$ of men and $41 \%$ of women had osteopenia based on BMD measurements before ICS initiation and did this not deteriorate and was comparable to placebo during the trial [18].

The only caution has been with pneumonia, where a slight increase in risk has been noted, but fortunately, no mortality [19]. The exact contribution of the ICS is perhaps debatable as COPD patients are known to be colonized with potential pathogens increasing their risk of pneumonia $a b$ initio.

\section{Phosphodiesterase inhibitors including Roflumilast}

Another pathway that has been exploited in COPD is the phosphodiesterase pathway. Clinicians are familiar with the phosphodiesterase inhibitor drug class in the example of theophylline (although it is equally well known that this inhibition does not occur with therapeutic doses in man and probably works via adenosine receptors). Theophylline has largely been used for its bronchodilator properties but does have other effects as well.

There are many phosphodiesterases but the PDE4 isoenzyme is the primary PDE expressed in inflammatory cells and airway smooth muscle [20]. PDE4 has a broad functional role in many inflammatory and immune cells including neutrophils that are central to inflammation and parenchymal changes and airway remodelling in COPD. A specific PDE4 inhibitor was identified, Roflumilast, studied extensively and has recently entered clinical practice (Daliresp). Research with this agent has shown that it is not a bronchodilator per se and is especially effective in severe COPD (FEV1 $\leq 50 \%$ ) with a dominant chronic bronchitis phenotype. The data on the agent show an improvement in pre and post-bronchodilator FEV1, decrease in the exacerbation rate and in some patient-reported outcomes [21, 22$]$.

Prevention of continual lung damage and lung function decline

The pathological features of chronic bronchitis and emphysema are the consequence of oxidant-antioxidant imbalance principally induced by smoking. It follows, therefore that smoking cessation is a most important and cardinal recommendation.

Conventional therapy-bronchodilators and ICS have anti-inflammatory properties and have been shown in longitudinal studies to have a minimal effect in decreasing the rate of lung function decline [6, 23, 24]. The question may well be asked-why do ICS not have a more obvious effect in improving lung function? It must be remembered that COPD constitutes major structural changes in the lungs and despite how powerful the anti-inflammatory effect, this is not going to regenerate lung tissue or improve lung function meaningfully - there will always be a physiological limit. This may perhaps explain the modest effects of the mitogen activated protein kinase (MAPK) inhibitors in COPD as well and indeed, equally unfortunately, for any new therapeutic modality [25].

Another factor contributing to accelerated lung function loss is an acute severe exacerbation. These life-threatening episodes, in addition to causing acute dysfunction and respiratory compromise, can aggravate lung damage and lead to sudden deterioration in the projected trajectory of age and smoking-accelerated lung function decrease [26].

\section{Decrease exacerbation risk}

Exacerbations are sustained deteriorations in respiratory symptoms that are frequently infective and thus place the COPD patient at considerable risk. When severe, they can be life threatening, usually requiring antibiotics, corticosteroid bursts, and hospitalization; thus they also represent a major cost driver in COPD. All drug classes have some effect on exacerbation frequency. In attempting to elucidate predisposing factors, a number of genetic and clinical studies have been performed, The Evaluation of COPD Longitudinally to Identify Predictive Surrogate Endpoints (ECLIPSE) study was able to show that exacerbators appeared to follow a pattern and that a clinical frequent exacerbator phenotype could be identified based simply on the history of exacerbations; this would be an important consideration and contributing factor in the revised guide [27••].

Other agents that have been studied that have shown a significantly decreased exacerbation risk include:

a) Carbocysteine - this is a mucolytic with anti-oxidant properties and in the PEACE study had a risk ratio of 0.75 (risk reduction: $95 \%$ CI 0.62-0.92) [28].

b) Azithromycin - this was investigated long term and achieved a risk ratio of 0.73 (risk reduction: $95 \% \mathrm{CI}$, 0.63 to 0.84 ) with a reduced time to first exacerbation; however, the impact of possibly increased antimicrobial resistance would need to be factored in [29].

Improvement in functional capacity

Pharmacological treatment has an important impact when it leads to improvements in patients' functional capacities and this has usually been evaluated using questionnaires - of which the St. George Respiratory Questionnaire (SGRQ) is the most validated and frequently used. Again all drug classes have had some effect with combined bronchodilator therapy and ICS having the best effect. However, all patients with COPD become deconditioned and pulmonary rehabilitation is 
extremely important to augment the effects of drug therapy. Improvement in static lung function alone, without rehabilitation, cannot improve the SGRQ substantially.

Decrease in mortality

The Holy Grail of COPD management is to improve mortality. Although all drugs classes have shown a tendency towards decreased mortality in the clinical trials, these have usually not reached statistical significance $[5,6]$.

\section{The new COPD guideline: what's different?}

There are a number of changes in the new GOLD guide. These help to better define and assist physicians in terms of the various aspects of COPD diagnosis and care. The principal changes are an appreciation of the need to decrease symptoms and to ameliorate factors that put patients at increased risk.

New definition

COPD is now defined as "a common preventable and treatable disease characterized by persistent airflow limitation that is usually progressive and associated with an enhanced chronic inflammatory response in the airways and the lung to noxious particles and gases. Exacerbations and co-morbidities contribute to the overall severity in individual patients."

In view of the confusion regarding bronchodilator responses as discussed earlier, this aspect of reversibility has been deleted.

Removal of stages

Previously, COPD was staged according to the numerical level of spirometric deficit. It has long been appreciated that spirometry is one dimensional and that there is in fact no correlation amongst the $\mathrm{FEV}_{1}$, functional capacity, and quality of life [30•]. The lung function measurement now simply falls into increasing categories of severity.

Introduction of new categorization

The multidimensional nature of the new guide serves to better encapsulate the various components of COPD into new categories. This is done by assessing and choosing 1 descriptor each from the 3 major facets of COPD that encompass symptoms and future risk:

i) Lung function measurement $\left(\mathrm{FEV}_{1}\right)$

ii) The degree of breathlessness

iii) The history of exacerbations

Lung function measurement ( $\left.F E V_{1}\right)$

The classification of the severity of airflow limitation utilizes the post-bronchodilator $\mathrm{FEV}_{1}$ expressed as a percentage of predicted and denoted as follows $[1 \bullet \bullet$ :

GOLD 1 (mild); post-bronchodilator predicted $\mathrm{FEV}_{1}$ : $\geq 80 \%$

GOLD 2 (moderate); post-bronchodilator predicted $\mathrm{FEV}_{1}: \geq 50 \%-<80 \%$

GOLD 3 (severe); post-bronchodilator predicted $\mathrm{FEV}_{1}$ : $\geq 30 \%-<50 \%$

GOLD 4 (very severe); post-bronchodilator predicted $\mathrm{FEV}_{1}:<30 \%$

However, it is a predictor of potential adverse events and is used to assess future risk.

\section{The degree of breathlessness}

Two scoring systems can be used to grade the degree of breathlessness:
Table 1 Diagrammatic representation of the 4 categories in the revised GOLD guideline and the appropriate therapeutic options $[1 \bullet \bullet$

*Derived from reference 1
Composite COPD categorization and treatment options*

\begin{tabular}{lll}
\hline Low risk & CATEGORY A & CATEGORY B \\
& MMRC: $0-1$ & MMRC: $2-4$ \\
& Airflow limitation: GOLD $1-2$ & Airflow limitation: GOLD $1-2$ \\
& Exacerbation history: $\leq 1 / \mathrm{yr}$ & Exacerbation history: $\leq 1 / \mathrm{yr}$ \\
& Therapy: Short/long acting bronchodilator & Therapy: Long acting bronchodilator \\
$\quad$ classes alone or in combination & classes alone or in combination \\
High risk & CATEGORY C & CATEGORY D \\
& MMRC: $0-1$ & MMRC: $2-4$ \\
& Airflow limitation: GOLD 3-4 & Airflow limitation: GOLD 3-4 \\
& Exacerbation history: $\geq 2 / \mathrm{yr}$ & Exacerbation history: $\geq 2 / \mathrm{yr}$ \\
& Therapy: Long acting bronchodilator classes & Therapy: Long acting bronchodilator \\
& alone or in combination \pm ICS & classes in combination \pm ICS
\end{tabular}


a) The COPD Assessment Test (CAT), an 8-item measurement of health status impairment in COPD (http:// catestonline.org).

b) The Modified British Medical Research Council (MMRC) Questionnaire that relates well to other measures of health status and is known to predict future mortality risk. For simplicity and because it is more user friendly, only this will be utilized for further stratification $[1 \bullet \cdot]$ :

Grade 0: I only get breathless with strenuous exercise Grade 1: I get short of breath when hurrying on the level or walking up a slight hill

Grade 2: I walk slower than people of the same age on the level because of breathlessness, or I have to stop for breath when walking on my own pace on the level

Grade 3: I stop for breath after walking about 100 meters or after a few minutes on the level

Grade 4: I am too breathless to leave the house or I am breathless when dressing or undressing

\section{The risk of exacerbations}

Utilizing the previous experience of the subjects, one fractionates them into those with less than or 2 or more exacerbations per year.

Having chosen one from each of the above domains, patients will now fall into one of 4 groups designated A, B (both lowrisk), and $\mathrm{C}$ and $\mathrm{D}$ (both high-risk) as illustrated in Table 1.

Therapeutic choices in each composite category

Bearing in mind the aforementioned discussion and subsequent categorization, the therapeutic choices can now be individualized. The first choice would be to select from the best available data and the most cost-effective options. If cost is not a major factor, long acting bronchodilators give the best symptomatic benefit. Category D represents the highest risk and the most symptomatic subjects; a variety of combinations based on physicians' experience, patient preferences and tolerance and cost may be selected. The alternative choices here are based on drug availability, cost, and the need for adjunctive therapy to optimize care. The options include theophylline, roflumilast, and carbocysteine. Oral corticosteroids are no longer recommended for the stable phase of COPD as they have a poor risk-benefit ratio: an inadequate functional response and a predisposition to the major adverse effects of steroids [31].

\section{Conclusions}

The new GOLD guide is a major departure from previous versions. Certain pillars of management-smoking cessation, pulmonary rehabilitation, and addressing comorbidities - are re-emphasized. The ability to make an accurate diagnosis rests on a careful history, clinical examination, and lung function testing. There are many pitfalls in spirometry, but one of the most important is to appreciate that many COPD patients do have significant reversibility and this should not compel one to make an erroneous diagnosis of asthma on that sole criterion. In assessing the COPD subject one makes a composite judgment based on spirometry, breathlessness, and the history of exacerbations. Thereafter, appropriately categorizing patients in terms of the need for symptomatic relief and the degree of risk allows one to select the best therapeutic option for optimal patient outcomes in the immediate and for the long term.

Acknowledgment The author gratefully acknowledges the GOLD scientific committee.

Disclosure E. M. Irusen: board membership (MSD, Novartis, GSK, Boehringer Ingelheim, AstraZeneca, Nycomed), consultancy (AstraZeneca, Boehringer Ingelheim, Nycomed), and speakers' bureaus (AstraZeneca, GSK, Nycomed, Novartis, MSD, Boehringer Ingelheim).

\section{References}

Papers of particular interest, published recently, have been highlighted as:

- Of importance

•- Of major importance

1. - Global Strategy for the Diagnosis, Management and Prevention of COPD, Global Initiative for Chronic Obstructive Lung Disease (GOLD) 2011. Available from: http://www.goldcopd.org/. (Accessed 4 June 2012). Excellent insight into the major literature that has informed the revision and the new individualized approach to management.

2. - Akinbami LJ, Moorman JE, Bailey C, et al. Trends in asthma prevalence, health care use, and mortality in the United States 2001-2010. NCHS Data Brief. 2012;94:1-8. Disadvantaged patients with poor access are the main group experiencing mortality.

3. Rabe K, Vermiere PA, Soriano JB, Maier WC. Clinical management of asthma in 1999: the Asthma Insights and Reality in Europe (AIRE) study. Eur Respir J. 2000;16:802-7.

4. - Bateman ED, Boushey HA, Bousquet J, et al. Can guidelinedefined asthma control be achieved? The Gaining Optimal Asthma ControL study. Am J Respir Crit Care Med. 2004;15:836-44. A landmark study on the utility of inhaled corticosteroid and longacting beta2-agonist fixed combinations versus ICS monotherapy in achieving asthma control.

5. Müller V, Gálffy G, Eszes N, et al. Asthma control in patients receiving inhaled corticosteroid and long-acting beta2-agonist fixed combinations. A real-life study comparing dry powder inhalers and a pressurized metered dose inhaler extrafine formulation. BMC Pulm Med. 2011;11:40.

6. Barnes PJ, Stockley RA. COPD: current therapeutic interventions and future approaches. Eur Respir J. 2005;25:1084-106.

7. •- Calverley PM, Anderson JA, Celli B, et al. Salmeterol and fluticasone propionate and survival in chronic obstructive 
pulmonary disease. N Engl J Med. 2007;356(8):775-89. A landmark study in COPD that offered many insights into the medium term course of $C O P D$.

8. Tashkin DP, Celli B, Senn S, et al. 4-year trial of tiotropium in chronic obstructive pulmonary disease. N Engl J Med. 2008;359 (15):1543-54

9. American Thoracic Society. Lung function testing: selection of reference values and interpretative strategies. Am Rev Respir Dis. 1991;144:1202-18.

10. Quanjer PH, Tammeling GJ, Cotes JE, et al. Lung volumes and forced ventilatory flows. Report Working Party Standardization of Lung Function Tests, European Community for Steel and Coal. Official Statement of the European Respiratory Society. Eur Respir J. 1993;6 Suppl 16:5-40.

11. Calverley PMA, Burge PS, Spencer S, Anderson JA, Jones PW. Bronchodilator reversibility testing in chronic obstructive pulmonary disease. Thorax. 2003;58:659-64.

12. - Richter DC, Joubert JR, Nell H, Schuurmans MM, Irusen EM. Diagnostic value of post-bronchodilator pulmonary function testing to distinguish between stable, moderate to severe COPD and asthma. Int J Chron Obstruct Pulmon Dis. 2008;3:693-9. Bronchodilator reversibility cannot differentiate between stable asthma and COPD.

13. Tashkin DP, Celli B, Decramer M, et al. Bronchodilator responsiveness in patients with COPD. Eur Respir J. 2008;31(4):742-50.

14. Barnes PJ, Ito K, Adcock IM. Corticosteroid resistance in chronic obstructive pulmonary disease: inactivation of histone deacetylase. Lancet. 2004;363:731-3.

15. Gan WQ, Man SF, Sin DD. Effects of inhaled corticosteroids on sputum cell counts in stable chronic obstructive pulmonary disease: a systematic review and a meta-analysis. BMC Pulm Med. 2005;5:3.

16. Barnes NC, Qiu YS, Pavord ID, et al. Antiinflammatory effects of salmeterol/fluticasone propionate in chronic obstructive lung disease. Am J Respir Crit Care Med. 2006;173:736-43.

17. Man SF, Zhang X, Vessey R, et al. The effects of inhaled and oral corticosteroids on serum inflammatory biomarkers in COPD: an exploratory study. Ther Adv Respir Dis. 2009;3:73-80.

18. Ferguson GT, Calverley PM, Anderson JA, et al. Prevalence and progression of osteoporosis in patients with COPD: results from the TOwards a Revolution in COPD Health study. Chest. 2009;136 (6):1456-65.
19. Crim C, Calverley PM, Anderson JA, et al. Pneumonia risk in COPD patients receiving inhaled corticosteroids alone or in combination: the TORCH study results. Eur Respir J. 2009;34:641-7.

20. Pryzwansky KB, Madden VJ. Type 4A cAMP-specific phosphodiesterase is stored in granules of human neutrophils and eosinophils. Cell Tissue Res. 2003;312:301-11.

21. - Rabe KF, Bateman ED, O'Donnell DWitte S, Bredenbroker D, Bethke TD. Roflumilast - an anti-inflammatory treatment for chronic obstructive pulmonary disease: a randomized controlled trial. Lancet. 2005;366:563-71. A 6-month study of the efficacy of roflumilast in moderate-to-severe COPD.

22. Calverley PM, Sanchez-Toril F, McIvor A, Teichmann P, Bredenbroeker D. Effect of 1-year treatment with roflumilast in severe chronic obstructive pulmonary disease. Am J Respir Crit Care Med. 2007;176:154-61.

23. Asano K, Shikama Y, Shoji N, et al. Tiotropium bromide inhibits TGF beta induced MMP production from lung fibroblasts by interfering with Smad and MAPK pathways in vitro. Int J Chron Obstruct Pulmon Dis. 2010;5:277-86.

24. Celli BR, Thomas NE, Anderson JA, et al. Effect of pharmacotherapy on rate of decline of lung function in chronic obstructive pulmonary disease: results from the TORCH study. Am J Respir Crit Care Med. 2008;178:332-8.

25. Chung KF. p38 mitogen-activated protein kinase pathways in asthma and COPD. Chest. 2011;139:1470-9.

26. Anzueto A. Impact of exacerbations on COPD. Eur Respir Rev. 2010;19:113-8.

27. •• Hurst JR, Vestbo J, Anzueto A, et al. Susceptibility to exacerbation in chronic obstructive pulmonary disease. N Engl J Med. 2010;363:1128-38. One of the publications from the ECLIPSE study which characterized the exacerbator phenotype.

28. Zheng JP, Kang J, Huang SG, et al. Effect of carbocisteine on acute exacerbation of chronic obstructive pulmonary disease (PEACE Study): a randomised placebo-controlled study. Lancet. 2008;371:2013-8.

29. Albert RK, Connett J, Bailey WC, et al. Azithromycin for prevention of exacerbations of COPD. N Engl J Med. 2011;365:689-98.

30. - Jones PW. Health status and the spiral of decline. COPD. 2009;6:59-63. A study demonstrating the poor relationship between lung function, functional capacity, and quality of life.

31. Walters JA, Walters EH, Wood-Baker R. Oral corticosteroids for stable chronic obstructive pulmonary disease. Cochrane Database Syst Rev. 2005;(3): CD005374. 